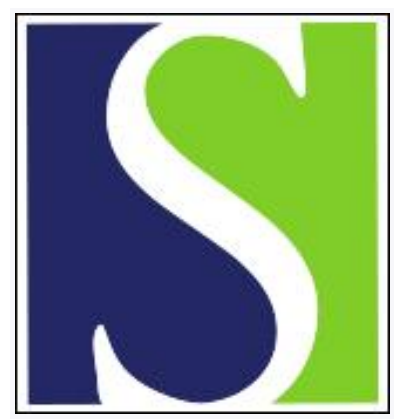

Scand J Work Environ Health 2015;41(1):5-15

https://doi.org/10.5271/sjweh.3462

Published online: 09 Oct 2014, Issue date: 01 Jan 2015

Asbestos, asbestosis, and cancer, the Helsinki criteria for diagnosis and attribution 2014: recommendations

by Wolff $\mathrm{H}$, reporter, Vehmas $\mathrm{T}$, reporter, Oksa P, reporter, Rantanen J, reporter, Vainio $\mathrm{H}$, reporter

Affiliation: Finnish Institute of Occupational Health ( $F I O H)$ Topeliuksenkatu 41aA 00250 Helsinki Finland. Henrik.Wolff@ttl.fi

Refers to the following texts of the Journal: 1997;23(4):311-316

2000;26(5):449-454

The following articles refer to this text: 2015;41(1):1-4;

2016;42(1):91-94; 2016;42(1):95-96

Key terms: asbestos; asbestosis; cancer; consensus report; exposure assessment; Helsinki criteria

This article in PubMed: www.ncbi.nlm.nih.gov/pubmed/25299403

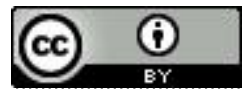




\section{Asbestos, asbestosis, and cancer, the Helsinki criteria for diagnosis and attribution 2014: recommendations}

\section{Introduction}

Although the use of asbestos has been banned in several industrialized countries, many workers continue to be exposed in asbestos repair and removal work, and asbestos is still widely used in various newly industrialized, rapidly developing countries. According to the most recent World Health Organization (WHO) estimates, more than 107000 people die each year from asbestos-related lung cancer, mesothelioma, and asbestosis resulting from exposure at work (1). The asbestos epidemic is far from over.

The expert meeting on "asbestos, asbestosis, and cancer" was convened in Helsinki in 1997 (2) and consisted of 19 participants from eight countries. This meeting had the goal to "discuss disorders in association with asbestos and agree upon state of the art criteria for diagnosis and attribution with respect to asbestos". In addition, questions concerning the surveillance of asbestos-exposed workers were discussed. The resulting consensus report was titled "Asbestos, asbestosis, and cancer: the Helsinki criteria for diagnosis and attribution" (in the current report a shorter name "Helsinki criteria" or just "criteria" will be used). A follow-up expert meeting on new advances in radiology and screening of asbestos-related diseases was organized in 2000 in Helsinki (3).

This report summarizes the results of a project, organized by the Finnish Institute of Occupational Health (FIOH), to update the 1997 and 2000 Helsinki criteria documents in view of the new advances in research. It presents the conclusions of a meeting on 10-13 February 2014 in Espoo, Finland, among an international group of experts working to update the criteria.

\section{Recommendations from the Helsinki criteria of 1997}

In the following, recommendations from the Helsinki criteria document of 1997 (and for radiology 2000) are quoted together with commentary by the authors.

\section{General considerations}

"In general, reliable work histories provide the most practical and useful measure of occupational asbestos exposure". "Using structured questionnaires and checklists, trained interviewers can identify persons who have a work history compatible with significant asbestos exposure." "A cumulative fibre dose expressed as fibreyears per cubic centimetre, is an important parametre of asbestos exposure."

"Analysis of lung tissue for asbestos fibres and asbestos bodies can provide data to supplement the occupational history."

"For clinical purposes, the following guidelines are recommended to identify persons with a high probability of exposure to asbestos dust:

- $\quad$ over 0.1 million amphibole fibres $(>5 \mu \mathrm{m})$ per gram of dry lung tissue or

- $\quad$ over 1 million amphibole fibres $(>1 \mu \mathrm{m})$ per gram of dry tissue as measured by electron microscopy in a qualified laboratory or

- over 1000 asbestos bodies per gram of dry tissue (100 asbestos bodies per gram of wet tissue) or

- over 1 asbestos body per millilitre of bronchoalveolar lavage fluid as measured by light microscopy in a qualified laboratory.

Each laboratory should establish its own reference values."

\section{Asbestosis}

"Asbestosis is defined as diffuse interstitial fibrosis of the lung as a consequence of exposure to asbestos dust." It is noted that neither clinical nor histological features of asbestosis differ sufficiently from those of other causes of interstitial fibrosis to allow confident diagnosis without a history of asbestos exposure or the detection of increased levels of asbestos bodies or asbestos fibres in lung tissue.

"Asbestosis is generally associated with relatively high exposure levels." It is however noted that mild fibrosis may occur at lower exposure levels and that histologically detectable fibrosis can occur in situations when radiological criteria are not fulfilled.

"A histological diagnosis of asbestosis requires the identification of diffuse interstitial fibrosis" in technically sound lung samples "plus the presence of either 2 
or more asbestos bodies in tissue with a section area of $1 \mathrm{~cm}^{2}$ or a count of uncoated asbestos fibres that falls in the range recorded for asbestosis by the same laboratory." It is also noted there is evidence that, in rare cases, asbestosis can occur without the presence of asbestos bodies and that such cases are only recognizable by uncoated fibre burden. In addition, it is noted there may be cases of chrysotile-induced asbestosis without increased levels of asbestos bodies or increased fibre burden, however, this is considered speculative.

"In order to achieve reasonable comparability between different studies a standardized system for the histological diagnosis and grading of asbestosis is required. The Roggli-Pratt modification of CAP-NIOSH system is recommended."

\section{Pleural disorders}

"Pleural plaques represent circumscribed areas of fibrous thickening typically of the parietal pleura." "In regions where plaques are not endemic, $80-90 \%$ of the plaques that are radiologically well-defined are attributable to occupational asbestos exposure."

"Diffuse pleural fibrosis designates non-circumscribed fibrous thickening of variable cellularity" involving mainly the visceral pleura. It "is probably the result of benign asbestos pleuritis with effusion. Diffuse pleural thickening can be associated with rounded atelectasis, it can be associated with mild or rarely moderate or severe restrictive pulmonary defects."

"Low exposures" from various sources "may induce pleural plaques. For diffuse pleural thickening higher exposure levels may be required".

\section{Mesothelioma}

"With the exception of certain histological types of mesothelioma that are benign or of an uncertain malignant potential (eg, multicystic mesothelioma, benign papillary mesothelioma), all types of malignant mesothelioma can be induced by asbestos with the amphiboles showing greater carcinogenic potency than chrysotile."

On requirements for and documentation of asbestos exposure to attribute causation:

- "A lung fibre count exceeding the background range for the laboratory in question." This is different and lower from the threshold value for persons with a high probability of exposure to asbestos dust at work mentioned earlier in the 'general considerations' paragraph.
- "or the presence of radiographic or pathological evidence of asbestos related tissue injury (e.g. asbestosis or pleural plaques)

- "or histopathological evidence of abnormal asbestos content (e.g. asbestos bodies in histological sections of lung) should be sufficient to relate a case of mesothelioma to asbestos exposure on a probability basis.

In the absence of such markers a history of significant occupational, domestic or environmental exposure will suffice for attribution. There is evidence that peritoneal mesotheliomas are associated with higher levels of asbestos exposure than pleural mesotheliomas."

"The following points need to be considered in the assessment of occupational aetiology:

- "The great majority of mesotheliomas are due to asbestos exposure;

- "Mesotheliomas can occur in cases with low asbestos exposure. However, very low background environmental exposures carry only an extremely low risk;

- "About $80 \%$ of mesothelioma patients have had some occupational exposure to asbestos, and therefore a careful occupational and environmental history should be taken;

- "Even a brief or low-level exposure should be considered sufficient for mesothelioma to be designated as occupationally related;

- "A minimum of 10 years from the first exposure is required to attribute the mesothelioma to asbestos exposure, though in most cases the latency interval is longer (eg, in the order of 30-40 years); and

- "Smoking has no influence on the risk of mesothelioma."

\section{Lung cancer}

Concerning types of lung cancer associated with asbestos exposure, "all four major histological types (squamous, adeno, large-cell and small cell) can be related to asbestos". In addition, location of the tumor in the lungs is not considered important in determining attributability.

"Attribution of causation requires a reasonable medical certainty on a probability basis that the agent (asbestos) has caused or contributed materially to the disease." Noting that increased exposure to asbestos also increases the risk, "cumulative exposure, on a probability basis should thus be considered the main criterion for the attribution of a substantial contribution by asbestos to lung cancer risk."

Using an estimate of $4 \%$ increase of risk for each fibres per cubic centimetre per year (fibre year) of cumulative 
exposure: "A cumulative exposure of 25 fibre-years is estimated to increase the risk of lung cancer 2-fold, clinical cases of asbestosis may occur at comparable cumulative exposures."

"A 2 fold risk of lung cancer is related to retained fibre levels of 2 million amphibole fibres $(>5 \mu \mathrm{m})$ per gram of dry lung tissue or 5 million amphibole fibres $(>1 \mu \mathrm{m})$ per gram of dry lung tissue. This lung fibre burden is approximately equal to 5000-15 000 asbestos bodies per gram of dry lung tissue, or 5-15 asbestos bodies per millilitre of bronchoalveolar lavage fluid." However, elsewhere in the consensus report, it is noted that each laboratory should establish its own reference values due to the lack of standardization of these methods.

"Occupational histories (fibre years of exposure) are probably a better indicator of lung cancer risk from chrysotile than fibre burden analysis" because of the higher clearance rates for chrysotile. "The presence of asbestosis is an indicator of high exposure. Asbestosis may also contribute some additional risk of lung cancer beyond that conferred by asbestos."

"Pleural plaques are indicators of exposure to asbestos fibres. Because pleural plaques may be associated with low levels of exposure, the attribution of lung cancer to asbestos must be supported by an occupational history of substantial asbestos exposure or measures of asbestos fibre burden," ie, pleural plaques alone are insufficient for the attribution of lung cancer to asbestos.

"Bilateral diffuse pleural thickening is often associated with moderate or heavy exposure, as seen in cases of asbestosis and should be considered accordingly in terms of attribution," ie, this type of pleural thickening is considered to be "close" to asbestosis in terms of exposure.

"A minimum lag time of ten years from the first asbestos exposure is required to attribute the lung cancer to asbestos."

"Not all exposure criteria need to be fulfilled for the purposes of attribution." For example, high fibre or asbestos-body counts with an uncertain work history should be considered.

"Although tobacco smoking affects the total lung cancer risk, this effect does not detract from the risk of lung cancer attributable to asbestos exposure. No attempt has been made in this report to apportion the relative contributions of asbestos exposure and tobacco smoking."
Radiology and screening, including follow-up meeting in 2000

For asbestosis diagnosis, the finding of International Labor Organization (ILO) profusion 1/0 was regarded as an early stage asbestosis and high-resolution computed tomography (HRCT) was recommended to be used as a diagnostic aid in selected cases in 1997.

In 2000, the technical requirements for asbestos HRCT were defined and the need for an international classification system for occupational HRCT findings led to a cooperative process between several investigators.

The issue of screening asbestos exposed workers for lung cancer remained open: research activity and the follow-up of ongoing investigations were encouraged.

\section{Recommendations}

To evaluate the need to update the Helsinki criteria, FIOH sent a questionnaire to selected international experts. In the questionnaire, we inquired about various aspects of the criteria, policies regarding the surveillance of asbestos-exposed workers, and experts' opinion on the need for and focus of updates to the criteria. The answers indicated a considerable variation of opinions although some general trends could be seen. The questionnaires, the possible updates, and the need for an update were discussed at an initial meeting of international experts and FIOH staff on 2 December 2011. After these discussions the following subject areas were selected for updating:

- CT screening for asbestos-related lung cancer;

- Follow-up of asbestos-exposed workers and diagnosis of non-malignant asbestos diseases;

- New asbestos-related disease entities; and

- Pathology and biomarkers.

For each subject area, a work-group prepared a review published separately. (4) Based on the reviews, recommendations for each subject area were made.

\section{Area 1. CT screening for asbestos-related lung cancer.}

It has been shown in the US national lung screening trial (NLST) (5) that lung cancer screening with low-dose computed tomography (LDCT) reduces both lung cancer mortality and over-all mortality among current and former smokers. Consequently, a growing number of organizations have issued recommendations to screen current or former (quit within 15 years) smokers in slightly varying age groups with LDCT (6-10). Some also endorse beginning of screening at age 50 years among adults with a 20-pack-year smoking history if they also have an additional risk factor, such as asbestos exposure, or if 
there is an additional cumulative risk of developing lung cancer of $\geq 5 \%$ in the next five years $(10,11)$

There are screening/surveillance programs of asbestos workers based on chest X-rays in some countries (12). This typically is a medicolegal program, and there is little scientific evidence that it is an effective strategy for reducing lung cancer mortality. There is increasing evidence from adults with significant smoking history that screening with LDCT reduces lung cancer mortality, but the generalizability to individuals at similar high risk based on asbestos exposure with or without smoking history is uncertain. Thus, we have two overarching recommendations for data gathering to evaluate these assumptions.

First, existing studies should be assessed for the potential of pooling to verify the generalizability of the LDCT random-controlled trial (RCT) results to asbestos-exposed high-risk populations. Second, since these updated Helsinki criteria recommendations are based on inferential evidence and modeling, the introduction of lung screening among asbestos-exposed workers should be accompanied by standardized data collection in order to acquire the necessary evidence to validate and refine these recommendations. Also, we strongly recommend the establishment of an international multicenter research project that could acquire these data for pooling, so that the specific effects of LDCT screening among asbestos-exposed workers can be measured and contribute to health policy. We believe the following study designs allow for the simultaneous enrollment of asbestos-exposed workers into a screening program, while also collecting minimum, but essential data. In settings:

(a) where LDCT is offered to current and former smokers at high risk, offer screening to high-risk workers with asbestos exposure with or without smoking history if the lung cancer risk is similar to the risk in high-risk smokers as defined by the NLST entry criteria and compare their outcomes with screened individuals at high risk based only on smoking history;

(b) that will demand evidence from RCT of asbestosexposed workers, conduct national or international pooled RCT;

(c) where LDCT screening is available for asbestos exposed workers but it is not offered to (a) current and former smokers at high risk in organized screening programs or (b) in RCT of asbestosexposed workers, follow standardized protocols from any one of the LDCT RCT to implement screening for asbestos-exposed workers who meet entry criteria based on asbestos exposure with or without tobacco exposure history [as defined in (a)] and monitor process and disease outcomes in comparison with respective RCT data for adults at risk for lung cancer based on smoking history alone.
In each of the examples above, the benefits, harms, and economic issues of LCDT screening should be studied. At this time, there is scant direct evidence on issues related to risk estimation and LDCT screening in workers at high risk for lung cancer due to asbestos exposure with or without a history of smoking. Based on the lung cancer LDCT screening studies, the dose-response risk of lung cancer in asbestos-exposed workers, and the established relationship of interaction of asbestos exposure and smoking, we recommend the following groups for LDCT screening:

- workers with any asbestos exposure and a smoking history equal to the entry criteria of the NLST study; and

- workers with asbestos exposure with or without a smoking history, which alone or together would yield an estimated risk level of lung cancer equal to that in the entry criteria of the NLST study.

Much work remains to be done related to risk estimation for lung cancer screening eligibility, especially the interplay between age, smoking history, other exposures to tobacco smoke, and other risk factors such as occupational history or genetic predisposition. In addition, the optimum screening interval should be further studied in forthcoming pilot trials with probably both annual and biennial arms included. Evidence may also be gained through modeling of existing materials, especially the NLST material.

Ideally, the responsibility for screening should be nationally or regionally organized so that preferably a single unit/institute would be in charge of the entire process. This includes the organization of screening, quality control, the collection and analysis of all data on benefits and complications as well as the evaluation of economic issues. The organizer may be the national occupational health institute, and it should have sufficient expertise on epidemiology, pulmonology, radiology and other relevant sciences. The participating units should be included in the most feasible manner nationally and educated in a sufficient manner in order to achieve high quality screening and follow-up and data collection. International collaboration is a high priority.

\section{Area 2. Follow-up of asbestos-exposed workers and diagnosis of non-malignant asbestos diseases}

Follow up of asbestos-exposed workers. The followup of asbestos-exposed workers has a long tradition from a preventive point of view and is mandatory in many countries. The recommended follow-up routines including both active and retired workers - differ among countries. Exposure history, disease history, pulmonary function and the chest radiograph have traditionally 
been the dominant methods. Computer tomography (CT) has during recent years been identified as useful in diagnosis and is emergent in screening of asbestosrelated diseases.

The traditional method for medical screening of asbestos-exposed workers has been the conventional chest radiograph, with standardized interpretation using the ILO system for classification of radiographs for pneumoconiosis (13). Radiographic surveillance as a part of periodical health examination (eg, every 3-5 years) of asbestos-exposed workers is still commonly practiced in developed countries. There is little specific scientific evidence of the health benefits of such surveillance. The potential benefits of the timely detection of non-malignant asbestos-related diseases includes the reduction in current asbestos exposure, an incentive to cease smoking, the encouragement of immunization and early treatment of respiratory infections, and increased health knowledge obtained by the participants. Those potential benefits must be weighed against the harm incurred as a result of the radiation dose received as part of radiographic surveillance.

The national recommendations to date have principally focused on non-malignant asbestos-related disease but may be developed at present to facilitate early detection of asbestos-related lung cancer by the use of CT. With regard to the use of CT for the early detection of lung cancer, a possible follow-up schema of asbestosexposed workers is presented in the recommendations from the Area 1 group.

With regard to the follow-up of non-malignant effects, the traditional guidelines vary widely between countries and are only to a limited degree based on scientific evidence. We still recommend that asbestos-exposed workers should be offered a medicolegal surveillance according to national regulations or compensation rules. These surveillance activities should when possible be organized as national programs and utilized for research.

A general follow-up schema of asbestos-exposed workers should be stratified according to the intensity, latency, and duration of exposure. In general, reliable work histories provide the most practical and useful measure of occupational asbestos exposure. High priority should be given to the high-risk groups, including retired workers.

Both radiographic and functional pulmonary deterioration (worsening) may occur long after asbestos exposure. Follow-up is necessary especially if it is relevant from a compensation point of view. We propose that the follow-up of highly asbestos-exposed workers should be continued for up to 30 years after the cessation of exposure.

In accordance with previous Helsinki criteria 1997 recommendations, we still recommend the use of spirometry together with questionnaires on past or current exposure, and current symptoms as a reference checkup for all asbestos-exposed workers. The questionnaire should provide detailed information on past (and current) asbestos exposure and smoking habits, specifically age of initiation, cigarettes per day, and date of cessation for former smokers. Especially if the follow-up schema is stratified according to the quantity of estimated exposure, it is recommended to calculate or approximate worker's exposure as fibre years if possible.

For clinical and medicolegal purposes, regular follow-up with spirometry, based on the national practice and legal requirements, is useful. A reasonable periodic interval is every $3-5$ years, dependent on past exposure level, time since cessation of exposure, and age. Measurements of carbon monoxide diffusion capacity might be used at baseline and in patients with documented asbestosis, but not for repeated screening purposes. It is important to advise all asbestos-exposed workers to contact their physician if they develop symptoms or signs of respiratory disease.

Biomarkers for follow-up and diagnostics. More studies are needed to assess if the markers of inflammation could be used to predict the risk of developing asbestosis in asbestos-exposed workers.

Immunization of asbestosis patients. We recommend influenza and pneumococcal vaccination to patients with asbestosis, even if there are no vaccination trials available (12).

Diagnosis of non-malignant asbestos diseases (asbestosis and pleural thickening)

The use of CT imaging in diagnosis of asbestos-related diseases may be useful when:

- a borderline finding of lung fibrosis (ILO 0/1$1 / 0$ ) is detected;

- there is a discrepancy between lung function finding of restriction and radiographs interpreted as normal;

- widespread pleural changes severely hamper the radiographic visibility of lung parenchyma.

CT imaging should be done using state-of-the-art multislice scanner technology and high resolution reconstruction algorithms. Exposure to ionizing radiation should be kept as low as possible.

The international expert meeting in Helsinki in 2000 recommended that a common, international classification scheme for pulmonary and pleural abnormalities detected on CT scans of asbestos-exposed workers should be established for the early identification of both malignant and non-malignant asbestos diseases, comparable to the 1980 ILO international classification 
of radiographs for pneumoconiosis (13). Such a common classification system is described in a monograph (14) and has been adopted by many countries. This classification has now been further developed to cover all types of occupational and environmental diseases (ICOERD) (15). Some countries such as France, have developed a nationally based classification system. For international comparison of studies of asbestos-exposed groups, we recommend use of the ICOERD classification.

Minimum criteria for diagnosis of asbestosis in CT. It is well known that small irregular opacities and pleural changes occur in the general population without work-related asbestos exposure. It is also well known that CT-detected lung fibrosis can be seen also in those with unlikely asbestos exposure and shows a distinct, although limited increase with age. The occurrence of limited lung fibrosis in the general population makes the definition of a threshold value for asbestosis important. We therefore recommend that the sum grade of $\geq 2-3$ bilateral irregular opacities in lower zones according to the reference film or bilateral honeycombing (sum grade $\geq 2$ ) would be sufficient to represent fibrosis according to the ICOERD system. In histopathology, bronchiolar wall fibrosis has been associated with asbestos exposure and other exposures including smoking (16). Subpleural curvilinear lines or dots in HRCT are findings of bronchiolar fibrosis.

Minimal pleural thickening. Very small pleural thickening is common and hard to separate from naturally thicker than average pleura, intercostal muscles, subpleural scars, adhesions etc ... in CT. According to the ICOERD, all visible pleural thickening is recorded: differential diagnosis of etiology depends upon disease and occupational history.

\section{Area 3. New asbestos-related disease entities}

Asbestos-related cancers. The legal standard of "more likely than not", equivalent to a relative risk (RR) of 2 , has been used in many countries as a threshold for attribution of causation of disease in individuals by hazardous exposures and was used as a threshold in the 1997 Helsinki criteria. However, the choice of a threshold for RR related to a hazardous exposure is based on social, economic, and political considerations, which vary between countries. In some situations, exposures associated with RR of as little as 1.1 have been viewed as making a material contribution to causation. We recommend that the threshold RR used for individual attribution should be no greater than 2 and can be set at lower levels. Whatever the threshold, it should be recognized that asbestos can contribute to causation of disease in exposed populations at even lower levels. To provide flexible guidance for setting threshold levels for individual causation, we have determined the relationship between RR for each reviewed new cancer entity and RR for lung cancer, using data from cohort studies that evaluated RR for both. The exposure needed to reach a threshold RR for the new entity cancer can then be determined based on that relationship. In our evaluation, this provided the most practical approach to providing criteria for individual attribution for these cancer entities.

Laryngeal cancer. Based on cohort studies evaluating standardized incidence ratios, the RR for laryngeal cancer in an asbestos-exposed cohort is somewhat less than the RR for lung cancer. The estimated RR based on cohort studies for laryngeal cancer would reach 2 under conditions where the RR for lung cancer in an exposed population was 2.8 (17). Standardized mortality ratios for the two cancer entities do not correlate well, perhaps in part because laryngeal cancer is less likely to result in mortality if appropriately treated and thus might be more variably captured on death certificates. Under conditions of asbestos exposure associated with an RR for lung cancer of 2, the estimated RR for laryngeal cancer is 1.6, with an estimated attributable fraction (AF) in the exposed population for asbestos-causation, of about $37 \%$.

The IARC has concluded (18) there is sufficient evidence for asbestos-causation of laryngeal cancer in humans, and the weight of litreature published after the IARC evaluation remains consistent with this evaluation. Thus, laryngeal cancer should be viewed as a disease that is caused by asbestos.

Ovarian cancer. The RR for ovarian cancer was slightly higher than the RR for lung cancer in an asbestosexposed cohort (17). The estimated RR for ovarian cancer would reach 2 under conditions where the RR for lung cancer in an exposed population was about 1.7. Under conditions of asbestos exposure associated with a RR for lung cancer of 2, the estimated RR for ovarian cancer is 2.2, with an estimated AF in the exposed population for asbestos-causation, of about $54 \%$.

The IARC has concluded that there is sufficient evidence for asbestos-causation of ovarian cancer in humans (18). The weight of the litreature remains consistent with the IARC's recent evaluation. Thus, ovarian cancer should be viewed as a disease that is caused by asbestos. Peritoneal mesothelioma should be considered in the differential diagnosis of women presenting with possible ovarian cancer who have been exposed to asbestos, since the two conditions can present similarly and be confused. Additional research is needed to document the specific histopathological types of ovarian cancer that are caused by asbestos exposure. 
Colorectal cancer. Few studies have determined risk estimates separately for colon and rectal cancer. Some data suggest that asbestos affects the risk of cancer of the colon, especially the proximal (right side) colon. The IARC's analysis of the relationship shows that in an asbestos-exposed cohort, the RR for colorectal cancer is much less than the RR for lung cancer (18). The estimated RR for colorectal cancer would reach 2 under conditions where the RR for lung cancer in an exposed population was about 5.2. Based on the IARC's analysis, under conditions of asbestos exposure associated with an RR for lung cancer of 2, the estimated RR for colorectal cancer is 1.1, with an estimated AF in the exposed population for asbestos-causation of colorectal cancer, of about $9 \%$.

The IARC has concluded that there is limited evidence in human epidemiology studies for an association between asbestos exposure and colorectal cancer in humans (18). The reports published since the IARC evaluation are not definitive. Recognizing that the available evidence is relatively strong, the weight of litreature remains consistent with the IARC's recent evaluation, and colorectal cancer cannot currently be viewed with certainty as a disease that is caused by asbestos.

Stomach cancer. In an asbestos-exposed cohort, the RR for stomach cancer is much less than the RR for lung cancer. The estimated RR for stomach cancer would reach 2 under conditions where the RR for lung cancer in an exposed population was approximately 4 (18). Under conditions of asbestos exposure associated with a RR for lung cancer of 2 , the estimated RR for stomach cancer is 1.2 , with an estimated AF in the asbestos-exposed population for asbestos-causation, of about $17 \%$.

IARC has concluded (18) that there is limited evidence in human epidemiology studies for an association between exposure to asbestos and stomach cancer in humans. Overall, most cohort studies and the various meta-analyzes provide consistent evidence of an increased risk of stomach cancer associated with asbestos exposure. Risk estimates tend to be higher in cohorts where heavy exposure to asbestos occurs and with long follow-up periods. There is also evidence that increasing exposure increases this risk. There are few case-control studies and these give less consistent results. The weight of the litreature remains consistent with the IARC's recent evaluation. Thus, stomach cancer cannot currently be viewed with certainty as a disease that is caused by asbestos.

Ventilatory impairment and chronic airway obstruction. Available studies indicate that the pattern of spirometric impairment associated with asbestos exposure is primarily restrictive, with reduced forced expiratory volume in one second $\left(\mathrm{FEV}_{1}\right)$, forced vital capacity (FVC), and relatively preserved $\mathrm{FEV}_{1} / \mathrm{FVC}$ ratio (17). Studies have also suggested that a component of airways obstruction can also be present, in particular small airways obstruction, although in the absence of other hazardous coexposures such as tobacco smoke, obstructive changes are generally of lesser prominence and more difficult to demonstrate. Statistically significant spirometric impairment can be demonstrated in populations with sufficient exposure to cause pleural thickening or asbestosis, even among those without radiographic changes. However, the prevalence of clinically significant spirometric impairment in individuals without asbestosrelated radiographic changes is unclear. The magnitude of impairment is considerably greater in the presence of diffuse pleural thickening (DPT) and/or asbestosis than if no asbestos-related radiographic changes are present. Also, some studies have shown that localized pleural thickening (LPT) can be associated with spirometric impairment. The magnitude of impairment increases with age and with smoking.

Findings of asbestos-induced pleuroparenchymal disease and emphysema have been reported to explain only about half the variance in total lung capacity (TLC), FVC, and carbon monoxide diffusing capacity (DLCO) among those exposed to asbestos with or without tobacco smoking. Therefore, more remains to be learned about the determinants of lung function impairment in asbestos-exposed individuals, particularly those without radiographic evidence of asbestos-induced parenchymal or pleural disease. In addition, more needs to be learned about how to attribute impairment in lung function to various potential causes when more than one potential cause is present.

Restrictive or mixed obstructive/restrictive patterns of ventilatory impairment associated with reduction of $\mathrm{FEV}_{1}$ below the lower limit of normal can be considered asbestos-caused if there has been asbestos exposure and radiographic pleural or parenchymal findings consistent with asbestos exposure are present. Ventilatory impairment of this type in the absence of asbestos-related radiographic changes cannot be viewed as asbestoscaused. Purely obstructive ventilatory impairment associated with reduction of $\mathrm{FEV}_{1}$ below the lower limit of normal cannot be viewed as being caused by asbestos.

Retroperitoneal fibrosis. Retroperitoneal fibrosis (RPF) is a rare disease featuring the proliferation of fibrous tissue in the retroperitoneal compartment of the body containing the kidneys, urinary tract, aorta, and various other structures. Evidence for causation of RPF by asbestos is suggested by a limited body of litreature including case reports, two case series and one case control study (17) Epidemiological evidence alone is insufficient to permit a firm conclusion about causation of RPF by asbestos. Still, taking the available case series into account, it is reasonable to conclude that 
RPF can be caused by asbestos. Those with sufficient exposure to develop asbestos-related pleural pathology are at increased risk. There is limited information about intensity of exposures required to cause RPF, relative potencies of different asbestos fibre types, etc.

RPF occurring in an individual with asbestos-related pleural and/or parenchymal radiographic findings can be viewed as caused by asbestos. RPF occurring in an individual with evidence of asbestos exposure, but without asbestos-related radiologic findings, can be viewed as caused by asbestos if other risk factors are not identified. All RPF patients should be evaluated for a history of asbestos exposure along with other risk factors.

\section{Area 4. Pathology and biomarkers}

Lung cancer types attributed to asbestos exposure. The 1997 Helsinki criteria mentions four major types of lung cancer that are associated with asbestos exposure (squamous, adeno, large-cell and small cell carcinoma). The current classification (19) mentions two additional types: sarcomatoid and adenosquamous carcinoma. Any of these six major histological categories may be considered to occur as a consequence of asbestos exposure (20).

Asbestosis: histological criteria. In the original 1997 Helsinki criteria, the Roggli-Pratt modification of the College of American Pathologists-National Institute of Occupational Safety and Health (CAP-NIOSH) classification for asbestosis was used. Recently, a new classification has emerged (16). In the new classification, bronchial fibrosis is designated "asbestos airways disease". The updated Helsinki criteria concerning the attributability of fibrotic conditions of the lungs to asbestos are applicable to both asbestosis as defined by the new classification and asbestos airways disease. The exposure levels for asbestos airways disease remain to be determined. A fibre analysis by an experienced laboratory is recommended in cases with idiopathic pulmonary fibrosis with a usual interstitial pneumonia (UIP) pattern, in which pleural plaques are present and/ or some asbestos bodies are present in histological sections (but fewer than $2 \mathrm{AB} / \mathrm{cm}^{2}$ ), or where compelling asbestos exposure history exists. In such cases, a fibreburden level comparable to those seen in that laboratory in other asbestosis cases favors a diagnosis of asbestosis.

Biomarkers for the histopathological diagnosis of malignant mesothelioma. We recommended that in mesotheliomas with an epithelioid component, at least two positive (mesothelial) and two negative (carcinomatous) markers be used for making a histopathological diagnosis of malignant mesothelioma. Because the usage of these markers has not yet been standardized, it is recommended that each laboratory performing immunohistochemical studies determine which positive and negative markers best fit its needs. It is further recommended that markers used should have $\geq 80 \%$ sensitivity and specificity.

In the case of pleural tumors the main differential diagnosis concerns pulmonary carcinomas. For peritoneal mesotheliomas, pulmonary carcinomas are much less likely to be in the differential diagnosis. The selection of negative markers should reflect this. It is also recommended that for peritoneal malignancies in women, stains for estrogen (ER) and progesterone (PR) receptors be added to the panel.

The diagnostic markers used in epithelioid mesotheliomas are far less useful in sarcomatoid mesotheliomas. Cytokeratin expression is a useful marker in the differentiation of desmoplastic mesothelioma from fibrotic processes (through demonstration of invasion) as well as differentiation of sarcomatoid mesothelioma from sarcomas. This marker is not useful in separating sarcomatoid mesotheliomas from sarcomatoid lung cancers.

It should be noted that clinical correlation with the gross distribution of the tumor is critical for diagnosis of malignant mesothelioma, and none of the immunohistochemical markers are entirely specific for that diagnosis. There are no generally accepted immunohistochemical markers for distinction between benign and malignant mesothelial proliferations.

Biomarkers for screening and early diagnosis of mesothelioma. Blood-borne biomarkers would arguably be ideal for screening purposes. Several markers have been studied with some success; however, currently none are sufficiently sensitive and specific for diagnostic or screening purposes.

A combination of biomarkers in pleural fluid is potentially useful for early diagnosis. A combination of CCL2, galectin-3, and soluble mesothelin-related peptides (SMRP) may discriminate between malignant mesothelioma patients and patients with metastatic adenocarcinoma or nonmalignant pleural effusion (21). Similarly, fluorescent in-situ hybridization (FISH) for homozygous deletion of p16 (INK2A) is a promising technique for distinguishing benign and malignant mesothelial pleural proliferations $(22,23)$.

Some of these markers may be useful in the treatment of malignancies as a follow-up tool and might help in early clinical diagnosis. A major unresolved question is whether early detection will improve treatment outcome. At this point, no specific recommendations can be made regarding these biomarkers for screening or other purposes.

Markers for attribution to asbestos exposure in lung cancer. The observed asbestos-related molecular alterations in lung cancer are consistent with the ability of asbestos fibres to induce DNA damage and chromosomal 
Table 1. Comparison of the 1997 Helsinki criteria and the 2014 update. [BAL=broncho alveolar lavage; CAP-NIOSH=College of American Pathologists-National Institute of Occupational Safety and Health ; CT=computer tomography; HRCT=high-resolution computer tomography; ICOERD=international classification of occupational and environmental disease; ILO=International Labor Organization; LDCT=Iowdensity computer tomography; WHO=World Health Organization.]

\begin{tabular}{|c|c|c|}
\hline Item & Helsinki criteria 1997 & Update 2014 \\
\hline \multirow{4}{*}{$\begin{array}{l}\text { General } \\
\text { considerations }\end{array}$} & \multirow{4}{*}{$\begin{array}{l}\text { Guidelines for identifying asbestos-exposed persons } \\
\text { with structured interview and fibers from tissue and } \\
\text { BAL specimen given. Guidelines for the diagnostics of } \\
\text { asbestosis, pleural disorders, mesothelioma and lung } \\
\text { cancer given. }\end{array}$} & Update concentrates on: \\
\hline & & $\begin{array}{l}\text { - } \quad \text { screening for asbestos-related lung cancer; } \\
\text { follow-up of asbestos-exposed workers and diagnosis of non-malignant } \\
\text { asbestos diseases; }\end{array}$ \\
\hline & & - $\quad$ new asbestos-related disease entities; and \\
\hline & & - $\quad$ pathology and biomarkers. \\
\hline
\end{tabular}

Asbestos-related Roggli-Pratt modification of the CAP-NIOSH classificanon-malignant tion of asbestosis recommended.

diseases Radiology: small opacities with ILO grade 1/0 in radiographs regarded as early stage asbestosis, HRCT in selected cases. Development of standardized reporting of HRCT scans recommended.

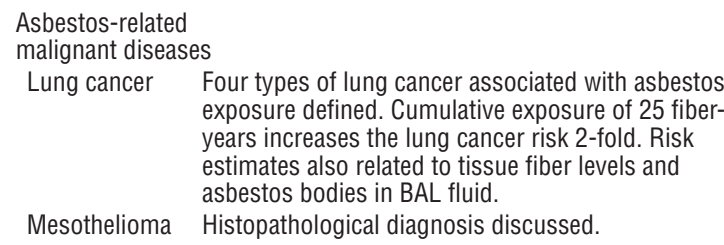

Mesothelioma Histopathological diagnosis discussed.

Other Discussed as research needs

malignancies

Surveillance and screening

Possibilities for primary and secondary prevention (screening) discussed. Scientific studies on screening recommended. Technical requirements for HRCT described (Helsinki conference in 2000).

Several research topics suggested.
New histology classification of asbestosis (16) is adapted.

Criteria for the use of CT imaging in the diagnostics of asbestos related diseases presented. Recommendation to use the international ICOERD CT classification in international studies.

Retroperitoneal fibrosis described as a new entity due to asbestos exposure (under certain conditions).

The current classification (WHO 1999) includes two additional types of lung cancer (sarcomatoid and adenosquamous). These are included as types of lung malignancies that may occur as a consequence of asbestos exposure.

Additional recommendations for histopathological diagnosis given for epithelioid and sarcomatoid mesotheliomas, separate recommendations for peritoneal mesotheliomas.

Laryngeal and ovarian cancers are included as cancers that may occur as a consequence of asbestos exposure. Guidelines for attribution given

Medicolegal surveillance (including spirometry) recommended according to the national regulation stratified according to the intensity, latency, and duration of exposure.

Vaccination against influenza and pneumococcus recommended for asbestosis patients.

LDCT screening recommended for asbestos-exposed workers under certain circumstances (see text for details). The importance of obtaining standardized data in an international setting is stressed. abnormalities. A combination of three chromosome abnormalities (2p16, 9p33.1, and 19p13) gave a clear dose-response between pulmonary fibre count and either allelic imbalance or copy number alteration or both in at least two of the regions, with a very high specificity when the three regions were combined (24). Additional international multicenter studies with standardized methodology for molecular assays and exposure assessment are necessary before these biomarkers can be applied to support causal attribution in individual cases.

\section{Epilogue}

The updating process was carried out in association with the International Conference of Monitoring and Surveillance of Asbestos-Related Diseases, 10-13 February, Espoo, Finland. The conference was organized by the
FIOH with $\mathrm{ICOH}$ as a co-organizer. The updates are based on the preparatory work of the four pre-meeting work groups. Their work will be published as reviews and is available on the FIOH website (4, 12, 17, 20, 25 ). In addition, the conference also made a declaration emphasizing the importance of primary prevention, regulation, health surveillance and diagnosis, and registration as well as research and collaboration on asbestos exposure and disease (26).

Much of the original criteria (2) remain unchanged although the Helsinki Criteria Update (HCU) 2014 group made some additions in specific issues, these can be seen in table 1 which summarizes the main points of the 1997 Helsinki criteria and its 2014 updates.

The updating process received grants from the Cancer Society of Finland, the Federation of Accident Insurance Institutions, the Federation of Finnish Learned Societies, and the Finnish Work Environment Fund which are gratefully acknowledged. 


\section{Participants}

Harri Vainio*, Finnish Institute of Occupational Health, Finland, Chairman HCU 2014, Panu Oksa*, Finnish Institute of Occupational Health, Finland, Vice Chairman HCU 2014, Sisko Anttila*, HUSLAB, Finland, Murray Finkelstein, University of Toronto, Canada, Heikki Frilander*, Finnish Institute of Occupational Health, Finland, Per Gustavsson, Karolinska Institutet, Sweden, Gunnar Hillerdal*, Karolinska University hospital, Sweden, Sergio Iavicoli*, INAIL, Italy, Kouki Inai*, Institute of Support Center of Remote Medicine, Japan, Agnes Kane*, Brown University, USA, Seong-Kyu Kang, Korea Occupational Safety and Health Agency, Republic of Korea, Takumi Kishimoto*, Okayama Rosai Hospital, Japan, Helge Kjuus*, National Institute of Occupational Health, Norway, Chairman Area 2, Thomas Kraus*, Aachen University, Germany, Yukinori Kusaka*, University of Fukui School of Medicine, Japan, Sverre Langård, Oslo University Hospital Ullevål, Norway, Nea Malila*, Finnish Cancer Register, Finland, Steven Markowitz*, City University of New York, USA, Anthony B Miller*, Canada, Aubrey Miller*, National Institute of Environmental Health Sciences, USA, Paula Pallasaho*, Finnish Institute of Occupational Health, Finland, John Parker, West Virginia University, USA, Jorma Rantanen*, Finland, Victor L Roggli*, Duke University Medical Center, USA, Chairman Area 4, Lesley Rushton, Imperial College London, UK, Riitta Sauni*, Ministry of Social Affairs and Health, Finland, Robert A Smith*, American Cancer Society, USA, Chairman Area 1, Leslie Stayner, University of Illinois at Chicago, USA, Kurt Straif*, IARC, France, Ken Takahashi*, University of Occupational and Environmental Health, Japan, Timo Tuomi*, Finnish Institute of Occupational Health, Finland, Axel Wannag*, Arbeidstilsynet (Dammen), Norway, Tapio Vehmas*, Finnish Institute of Occupational Health, Finland, David N. Weissman*, National Institute for Occupational Safety and Health, USA, Chairman Area 3, Henrik Wolff*, Finnish Institute of Occupational Health, Finland

*Present at update meeting in Helsinki 10-13 February 2014.

\section{Acknowledgment}

At the beginning of the meeting for updating the criteria, declarations of interest information were requested and obtained from all the participants. Most had nothing to declare with the following exceptions: Murray Finkelstein has provided expert opinion as a consultant in American asbestos litigation; Thomas Kraus has provided expert opinion in medico-legal cases involv- ing asbestos-related diseases; Steven Markowitz has provided expert opinion in medico-legal cases involving asbestos-related diseases and LDCT scanning for lung cancer screening; Victor L Roggli has had his travel expenses paid to attend and present at Defense Research Institute and Perrin asbestos conferences, and he has consulted as an expert witness for plaintiff and defense firms in asbestos litigation; Leslie Stayner has provided expert opinion as a consultant in medico-legal cases of workers who have developed mesothelioma from occupational exposure to asbestos; Kurt Straif is a member of the IARC Asbest Study team. The study is funded by the Ministry of Health of the Russian Federation, approved by the IARC Ethics Committee and monitored by an independent Scientific Advisory Board; Axel Wannag has worked as an occupational physician for the Norwegian asbestos cement industry in the past (closed 1981).

\section{References}

1. World Health Organization (WHO). Asbestos: elimination of asbestos-related diseases. Fact sheet no. 343. Paris:WHO; 2010. Available from: http://www.who.int/mediacentre/ factsheets/fs343/en/index.html.

2. Tossavainen A Asbestos, asbestosis, and cancer: the Helsinki criteria for diagnosis and attribution. Scand J Work Environ Health. 1997;23(4):311-6. http://dx.doi.org/10.5271/ sjweh. 226

3. Tossavainen A. International expert meeting on new advances in the radiology and screening of asbestos-related diseases. Scand J Work Environ Health. 2000;26(5):449-54. http:// dx.doi.org/10.5271/sjweh.567

4. Asbestos Asbestosis and Cancer, the Helsinki Criteria for Diagnosis and Attribution 2014. Helsinki: Finnish Institute of Occupational Health; 2014. Available from: www.ttl.fi/ hcuasbestos.

5. National Lung Screening Trial Research Team. Reduced lung-cancer mortality with low-dose computed tomographic screening. N Engl J Med. 2011;365(5):395-409. http://dx.doi.org/10.1056/NEJMoa1102873

6. American Lung Association. Providing guidance on lung cancer screening to patients and physicians. Washington DC: American Lung Association; 2012. Available from: http:// www.lung.org/lung-disease/lung-cancer/lung-cancerscreening-guidelines/lung-cancer-screening.pdf.

7. Wender R, Fontham ET, Barrera E, Jr., Colditz GA, Church TR, Ettinger DS, et al. American Cancer Society lung cancer screening guidelines. CA Cancer J Clin. 2013;63(2):107-17. http://dx.doi.org/10.3322/caac.21172

8. Detterbeck FC, Mazzone PJ, Naidich DP, Bach PB. Screening for lung cancer: Diagnosis and management of lung cancer, 3rd ed: American College of Chest Physicians evidence-based clinical practice guidelines. Chest. 2013;143(5 Suppl):e78S92S. 
9. Moyer VA. Screening for lung cancer: U.S. Preventive Services Task Force recommendation statement. Ann Intern Med. 2014;160(5):330-8. http://dx.doi.org/10.7326/M132771

10. Jaklitsch MT, Jacobson FL, Austin JH, Field JK, Jett JR, Keshavjee S, et al. The American Association for Thoracic Surgery guidelines for lung cancer screening using low-dose computed tomography scans for lung cancer survivors and other high-risk groups. J Thorac Cardiovasc Surg. 2012;144(1):33-8. http://dx.doi.org/10.1016/j.jtcvs.2012.05.060

11. Wood DE, Eapen GA, Ettinger DS, Hou L, Jackman D, Kazerooni E, et al. Lung cancer screening. J Natl Compr Canc Netw. 2012;10(2):240-65.

12. Kjuus H, Vehmas T, Finkelstein M, Kraus T, Kishimoto T, Frilander H, et al. Follow-up of asbestos exposed workers and diagnosis of non-malignant asbestos diseases. Helsinki: Finnish Institute of Occupational Health; 2014. Available from: www.ttl.fi/hcuasbestos.

13. International Labor Organization (ILO). Guidelines for the use of the ILO international classification of radiographs of pneumoconioses. Geneva: ILO; Revised edition 1980/2000

14. Kusaka Y, Hering KG, Parker JE. International Classification of HRCT for occupational and enviromental diseases. Tokyo: Springer; 2005.

15. Suganuma N, Kusaka Y, Hering KG, Vehmas T, Kraus T, Arakawa $\mathrm{H}$, et al. Reliability of the proposed international classification of high-resolution computed tomography for occupational and environmental respiratory diseases. J Occup Health. 2009;51(3):210-22. http://dx.doi.org/10.1539/joh. L8030

16. Roggli VL, Gibbs AR, Attanoos R, Churg A, Popper H, Cagle P, et al. Pathology of asbestosis- An update of the diagnostic criteria: Report of the asbestosis committee of the college of American pathologists and pulmonary pathology society. Arch Pathol Lab Med. 2010;134(3):462-80.

17. Weissman D, Gustavsson P, Miller A, Rushton L, Stayner L, Pallasaho P, et al. New Asbestos Related Disease Entities. Helsinki: Finnish institute of Occupational Health; 2014. Available from: www.ttl.fi/hcuasbestos.

18. A review of human Carcinogens. Arsenic, Metals, Fibres and Dust. IARC Monographs on the Evaluation of the Carcinogenic Risks to Humans. 100 part C. Lyon: IARC; 2012.

19. Travis W, Colby TV, Corrin B, Shimosato Y, Brambilla E. The World Health Organization Histological Typing of Lung and Pleural Tumours. 3 rd ed. Berlin: Springer Verlag; 1999.
20. Roggli VL, Anttila S, Kane A, Inai K, Wolff H. Pathology and Biomarkers. Helsinki: Finnish Institute of Occupational Health; 2014. Available from: www.ttl.fi/hcuasbestos.

21. Blanquart C, Gueugnon F, Nguyen JM, Roulois D, Cellerin L, Sagan C, et al. CCL2, galectin-3, and SMRP combination improves the diagnosis of mesothelioma in pleural effusions. J Thorac Oncol. 2012;7(5):883-9. http://dx.doi.org/10.1097/ JTO.0b013e31824c9272

22. Savic S, Franco N, Grilli B, Barascud Ade V, Herzog M, Bode $\mathrm{B}$, et al. Fluorescence in situ hybridization in the definitive diagnosis of malignant mesothelioma in effusion cytology. Chest. 2010;138(1):137-44.

23. Han J, Cao S, Zhang K, Zhao G, Xin Y, Dong Q, et al Fluorescence in situ hybridization as adjunct to cytology improves the diagnosis and directs estimation of prognosis of malignant pleural effusions. J Cardiothorac Surg. 2012;7:121. http://dx.doi.org/10.1186/1749-8090-7-121

24. Nymark P, Aavikko M, Makila J, Ruosaari S, HienonenKempas T, Wikman H, et al. Accumulation of genomic alterations in $2 \mathrm{p} 16,9 \mathrm{q} 33.1$ and $19 \mathrm{p} 13$ in lung tumours of asbestos-exposed patients. Mol Oncol. 2013;7(1):29-40. http:// dx.doi.org/10.1016/j.molonc.2012.07.006

25. Vehmas T, Sauni R, Miller AB, Straif K, Malila N, Smith RA. Screening for asbestos-related lung cancer. Helsinki Finnish Institute of Occupational Health; 2014. Available from: www. ttl.fi/hcuasbestos

26. The Helsinki Declaration on Management and Elimination of Asbestos-Related Diseases. Available from: http://www. icohweb.org/site_new/ico_news_detail.asp?id=86.

Report compiled by:

Henrik Wolff, MD, PhD ${ }^{1}$

Tapio Vehmas, MD, PhD ${ }^{1}$

Panu Oksa, MD, PhD 1

Jorma Rantanen, MD, PhD, ${ }^{2}$

Harri Vainio, MD, PhD ${ }^{1}$

on behalf of the Helsinki Criteria Updating Group.

${ }^{1}$ Finnish Institute of Occupational Health (FIOH), Helsinki, Finland

${ }^{2}$ International Commission on Occupational Health (ICOH), Helsinki, Finland

Correspondence to: Dr. Henrik Wolff, Finnish Institute of Occupational Health (FIOH) Topeliuksenkatu 41aA 00250 Helsinki Finland. [E-mail Henrik.Wolff@ttl.fi] 